Article

\title{
Ordinary Differential Equation with Left and Right Fractional Derivatives and Modeling of Oscillatory Systems
}

\author{
Liana Eneeva *(D), Arsen Pskhu (1) and Sergo Rekhviashvili \\ Institute of Applied Mathematics and Automation, Kabardino-Balkarian Scientific Center of Russian \\ Academy of Sciences, 89-A Shortanov Street, 360000 Nalchik, Russia; pskhu@list.ru (A.P.); rsergo@mail.ru (S.R.) \\ * Correspondence: eneeva72@list.ru;
}

Received: 30 October 2020; Accepted: 26 November 2020; Published: 27 November 2020

\begin{abstract}
We consider the principle of least action in the context of fractional calculus. Namely, we derive the fractional Euler-Lagrange equation and the general equation of motion with the composition of the left and right fractional derivatives defined on infinite intervals. In addition, we construct an explicit representation of solutions to a model fractional oscillator equation containing the left and right Gerasimov-Caputo fractional derivatives with origins at plus and minus infinity. We derive a representation for the composition of the left and right derivatives with origins at plus and minus infinity in terms of the Riesz potential, and introduce special functions with which we give solutions to the model fractional oscillator equation with a complex coefficient. This approach can be useful for describing dissipative dynamical systems with the property of heredity.
\end{abstract}

Keywords: principle of least action; fractional oscillator; equation with left and right fractional derivative; Gerasimov-Caputo fractional derivative; Riesz potential

MSC: $34 \mathrm{~A} 08 ; 70-10$

\section{Introduction}

The basic framework for Lagrangian and Hamiltonian mechanics is the principle of least action. In problems of mechanics, the principle of stationary action is most commonly used, and is the most important among all extreme principles. The equations of motion obtained using these principles are called the Euler-Lagrange equations. The Euler-Lagrange equation with the Lagrange function can describe any mechanical motion.

In recent years, fractional integro-differentiation has been widely used to model the properties of dynamic systems [1-3]. Fractional calculus enables one to naturally take into account the heredity, time non-locality and memory effects in dynamic processes [2].

The principle of least action in the context of the fractional calculus is studied in [4-11]. In [4,5], it was shown that applying the Lagrangian with fractional derivatives allows one to describe the dynamics of nonconservative systems. In [6], two fractional variational problems were considered. A generalized Euler-Lagrange equation with fractional differentiation was derived. The classical Euler-Lagrange equation is a special case of the latter. In addition, practical examples of the application of the results obtained were considered. It was shown that this approach would make it possible to simulate dynamic systems with greater accuracy. In [8], the Lagrange formalism with fractional derivatives with infinite limits was considered for the first time. In [9], the Hamiltonian and Lagrangian formulations with fractional differentiation were used to describe constrained systems. It was shown that both treatments for systems with linear velocities are equivalent. In $[7,12,13]$ the principle resulted 
in very exotic differential equations of fractional order, with composition of the Riemann-Liouville or Caputo operators with different origins. These equations and their possible application to modeling dynamic processes can be found in [12-17].

We point out the major challenges related to the equations with different origins. First, methods for obtaining solutions to these equations, both analytically and numerically, are not yet well developed. Next, physical interpretation of the fractional Euler-Lagrange equations is currently not well understood. The first-order Euler-Lagrange equation does not contain the limits of integration in the action functional, unlike the fractional Euler-Lagrange equation. Therefore, the following question arises: is it possible to use a fractional Euler-Lagrange equation if the time variable falls outside the interval within which the action functional is considered? Any value can be chosen as a time interval, including a small physical time (for example, the Planck time, which is approximately $5.4 \times 10^{-44} \mathrm{~s}$ ). In this case, studying any physical process in this interval is believed to be incorrect.

This ambiguity in a sense can be eliminated by using the Gerasimov-Caputo fractional derivatives (fractional operators defined on an infinite interval $[18,19]$ ) in the Euler-Lagrange equation, and we offer this in the present article.

In the present paper, we derive the fractional Euler-Lagrange equation and the general equation of motion with the composition of the left and right fractional derivatives defined on infinite intervals, and construct an explicit representation of solutions to a model fractional oscillator equation containing the left and right Gerasimov-Caputo fractional derivatives with origins at plus and minus infinity.

The rest of the paper is structured as follows: Section 2 contains the definitions of fractional integrals and derivatives; in Section 3, we derive the Euler-Lagrange equation and the equation of motion with fractional derivatives defined on infinite intervals; and, in Section 4, we construct solutions of a model one-dimensional equation for the fractional oscillator; Section 5 presents the main findings of this work.

\section{Fractional Differentiation}

The fractional derivatives of order $\alpha(0<\alpha<1)$ with respect to $t$, having origins at $t=a$ $(-\infty \leq a \leq \infty)$, in the Riemann-Liouville and Gerasimov-Caputo senses, are given by ([18-21])

$$
D_{a t}^{\alpha} g(t)=\operatorname{sign}(t-a) \frac{d}{d t} D_{a t}^{\alpha-1} g(t) \quad \text { and } \quad \partial_{a t}^{\alpha} g(t)=\operatorname{sign}(t-a) D_{a t}^{\alpha-1} \frac{d}{d t} g(t),
$$

respectively. Here, $D_{a t}^{\alpha-1}$ stands for the Riemann-Liouville fractional integral:

$$
D_{a t}^{\alpha-1} g(t)=\operatorname{sign}(t-a) \int_{a}^{t} g(\eta) \frac{|t-\eta|^{-\alpha}}{\Gamma(1-\alpha)} d \eta
$$

\section{Euler-Lagrange Equation}

Introduce a velocity vector for a material point motion in a certain medium:

$$
\mathbf{v}(t)=\frac{1}{\tau \Gamma(1-\alpha)} \int_{-\infty}^{t}(t-\eta)^{-\alpha} \frac{d}{d \eta} \mathbf{r}(\eta) d \eta=\frac{1}{\tau} \partial_{-\infty t}^{\alpha} \mathbf{r}(t) \quad(0<\alpha \leq 1),
$$

where $\mathbf{r}(t)$ is the radius vector, $t$ is the dimensionless (related to $\tau$ ) time and $\tau$ is some characteristic time for the process. On account of Equation (1), the generalized action functional can be written as

$$
S=\int_{-\infty}^{\infty} L[t, \mathbf{r}(t), \mathbf{v}(t)] d t
$$

In Equation (2) infinite limits for integration are established. To determine the velocity vector of Equation (1), it is necessary to take into account the entire time axis. Assume $\mathbf{r}_{0}(\mathbf{t})$ is a solution for the extremum problem. Then by Equation (2) and the conditions 


$$
\begin{aligned}
& \mathbf{r}(t)=\mathbf{r}_{0}(t)+\varepsilon \mathbf{h}(t), \\
& \mathbf{h}(-\infty)=\mathbf{h}(\infty)=0
\end{aligned}
$$

( $\varepsilon$ is a small parameter) we get

$$
S=\int_{-\infty}^{\infty} L\left[t, \mathbf{r}_{0}(t)+\varepsilon \mathbf{h}(t), \frac{1}{\tau} \partial_{-\infty t}^{\alpha} \mathbf{r}_{0}(t)+\frac{\varepsilon}{\tau} \partial_{-\infty t}^{\alpha} \mathbf{h}(t)\right] d t .
$$

The extremum of the functional satisfies the condition

$$
\frac{d S}{d \varepsilon}=\int_{-\infty}^{\infty}\left(\frac{d L}{d \varepsilon}\right) d t=\int_{-\infty}^{\infty} \mathbf{h}(t)\left(\frac{\partial}{\partial \mathbf{r}}+\frac{1}{\tau} D_{\infty t}^{\alpha} \frac{\partial}{\partial \mathbf{v}}\right) L[t, \mathbf{r}(t), \mathbf{v}(t)] d t=0 .
$$

Using Equation (3) we obtain the Euler-Lagrange equation

$$
\left(\frac{\partial}{\partial \mathbf{r}}+\frac{1}{\tau} D_{\infty t}^{\alpha} \frac{\partial}{\partial \mathbf{v}}\right) L[t, \mathbf{r}(t), \mathbf{v}(t)]=0
$$

It should be noted that the same problem for classical fields was first considered in [8]. In our case, Equation (4) defines the action function for a mechanical system. Moreover, the other techniques can be applied to get Equation (4), for example, using the results of [22], obtained by exploiting the Riesz fractional derivative.

Next, assume that the space of the considered motion is homogeneous and isotropic. Then the scalar function $L$ should not be explicitly dependent on time. The function $L$ should undergo power series expansion that involves only even powers $\mathbf{v}(t)$. Up to the second term of the expansion, we have

$$
L=\frac{m \mathbf{v}^{2}(t)}{2}-U[\mathbf{r}(t), t] .
$$

Expansion coefficients $m$ and $U[\mathbf{r}(t), t]$ are independent of the derivative fractional order $\alpha$. Therefore, there is no indication that these coefficients are different from the mass and potential energy of an object. Substituting Equation (5) into Equation (4), we obtain the equation of motion

$$
-\frac{m}{\tau^{2}} D_{\infty t}^{\alpha} \partial_{-\infty t}^{\alpha} \mathbf{r}(t)=\mathbf{F}[\mathbf{r}(t), t],
$$

where $\mathbf{F}[\mathbf{r}(t), t]=-(\partial / \partial \mathbf{r}) U[\mathbf{r}(t), t]$. The right-hand side of Equation (6) does not explicitly depend on $\alpha$, and, consequently, $\mathbf{F}[\mathbf{r}(t), t]$ determines the conservative force acting on a material point. The parameter $\alpha$, according to $[23,24]$ takes into account energy dissipation. Therefore, the equation of motion in the form of Equation (6) is apparently obtained for the first time.

As $\alpha=1$, by Equation (6) we have the classical equation of motion

$$
\frac{m}{\tau^{2}} \frac{d^{2} \mathbf{r}(t)}{d t^{2}}=\mathbf{F}[\mathbf{r}(t), t] .
$$

\section{Solution of Fractional Oscillator Equation}

Here, inspired by Equation (6), we are going to solve the equation

$$
D_{\infty t}^{\alpha} \partial_{-\infty t}^{\alpha} x(t)+\lambda x(t)=f(t) \quad(\lambda \in \mathbb{C}),
$$

which is a model one-dimensional equation for the fractional oscillator.

Definition 1. By a solution of Equation (7), we mean a function $x(t)$ such that $x^{\prime}(t) \in L^{1}(\mathbb{R})$, $D_{\infty t}^{\alpha-1} D_{-\infty t}^{\alpha-1} x(t) \in C^{1}(\mathbb{R})$ and $x(t)$ satisfies Equation (7). 


\subsection{Riesz Potentials}

Now, we will rewrite Equation (7) in terms of the Riesz potential.

Definition 2. Let $\mu \in(0,1)$. The operator $K^{\mu}$ is called the Riesz potential, and, for an integrable function $x(t)$, it is defined by

$$
K^{\mu} x(t)=\left(K^{\mu} x\right)(t)=c_{\mu} \int_{-\infty}^{\infty}|t-s|^{\mu-1} x(s) d s, \quad c_{\mu}=\frac{\Gamma(1-\mu)}{\Gamma\left(\frac{\mu}{2}\right) \Gamma\left(1-\frac{\mu}{2}\right)} .
$$

Lemma 1. Let $\mu \in(0,1 / 2)$. If $x(t) \in L^{1}(\mathbb{R})$, then

$$
D_{\infty t}^{-\mu} D_{-\infty t}^{-\mu} x(t)=K^{2 \mu} x(t)
$$

Proof. Let $M=\max \{s, t\}$ and $m=\min \{s, t\}$. It is easy to check that

$$
\begin{gathered}
{[\Gamma(\mu)]^{2} D_{\infty t}^{-\mu} D_{-\infty t}^{-\mu} x(t)=\int_{t}^{\infty}(\xi-t)^{\mu-1} \int_{-\infty}^{\xi}(\xi-s)^{\mu-1} x(s) d s d \xi} \\
=\int_{-\infty}^{\infty} x(s) \int_{-\infty}^{m}(M-\xi)^{\mu-1}(m-\xi)^{\mu-1} x(s) d \xi d s \\
=\int_{-\infty}^{\infty} x(s) \int_{0}^{\infty}(M-m+\eta)^{\mu-1} \eta^{\mu-1} d \eta d s \\
=\int_{-\infty}^{\infty} x(s)(M-m)^{2 \mu-1} d s \cdot \int_{0}^{\infty}(1+z)^{\mu-1} z^{\mu-1} d z .
\end{gathered}
$$

Combining this with the relations

$$
M-m=|t-s| \quad \text { and } \quad \int_{0}^{\infty}(1+z)^{\mu-1} z^{\mu-1} d z=\frac{\Gamma(\mu) \Gamma(1-2 \mu)}{\Gamma(1-\mu)},
$$

we get Equation (8).

By Equation (8) we have

$$
D_{\infty t}^{\alpha} \partial_{-\infty t}^{\alpha} x(t)=-\frac{d}{d t} D_{\infty t}^{\alpha-1} D_{-\infty t}^{\alpha-1} \frac{d}{d t} x(t)=-\frac{d}{d t} K^{2-2 \alpha} \frac{d}{d t} x(t),
$$

and, consequently, Equation (7) can be rewritten in the form

$$
-\frac{d}{d t} K^{2-2 \alpha} \frac{d}{d t} x(t)+\lambda x(t)=f(t) .
$$

\subsection{Special Functions}

Consider the functions

$$
C_{\alpha, \lambda}(t)=\frac{1}{\pi} \int_{0}^{\infty} \frac{\cos \xi t}{\xi^{2 \alpha}+\lambda} d \xi
$$

and

$$
S_{\alpha, \lambda}^{\mu}(t)=\frac{1}{\pi} \int_{0}^{\infty} \frac{\xi^{\mu} \sin \xi t}{\xi^{2 \alpha}+\lambda} d \xi
$$

for $t \in \mathbb{R}, \lambda \in \mathbb{C} \backslash\{0\}, \alpha \in(1 / 2,1)$ and $\mu \in(-2,2 \alpha)$.

Lemma 2. Let $\lambda \in \mathbb{C} \backslash\{0\}$ and $\alpha \in(1 / 2,1)$. Then

$$
\frac{d}{d t} C_{\alpha, \lambda}(t)=-S_{\alpha, \lambda}^{1}(t), \quad \frac{d}{d t} S_{\alpha, \lambda}^{-1}(t)=C_{\alpha, \lambda}(t),
$$


and

$$
K^{2-2 \alpha} S_{\alpha, \lambda}^{1}(t)=\frac{\operatorname{sign} t}{2}-\lambda S_{\alpha, \lambda}^{-1}(t)
$$

Moreover, if $|\arg \lambda|<(1-\alpha) \pi-\varepsilon$ for some $\varepsilon>0$, then

$$
\lim _{|t| \rightarrow \infty} t^{2} C_{\alpha, \lambda}(t)<\infty \quad \text { and } \quad \lim _{|t| \rightarrow \infty} t^{3} S_{\alpha, \lambda}^{1}(t)<\infty .
$$

Proof. The formulas in Equation (9) are derived by direct differentiation. In the same way, an easy computation gives

$$
\begin{gathered}
K^{2-2 \alpha} S_{\alpha, \lambda}^{1}(t)=S_{\alpha, \lambda}^{2 \alpha-1}(t) \\
=\frac{1}{\pi} \int_{0}^{\infty} \frac{\xi^{2 \alpha-1} \sin \xi t}{\xi^{2 \alpha}+\lambda} d \xi=\frac{1}{\pi} \int_{0}^{\infty} \frac{\sin \xi t}{\xi} d \xi-\lambda S_{\alpha, \lambda}^{-1}(t) .
\end{gathered}
$$

This proves Equation (10).

Using the properties of the Mittag-Leffler function (see e.g., [25]), we can write

$$
\begin{gathered}
C_{\alpha, \lambda}(t)=\frac{1}{\pi} \int_{0}^{\infty} \cos \xi t \int_{0}^{\infty} e^{-s \xi} s^{2 \alpha-1} E_{2 \alpha, 2 \alpha}\left(-\lambda s^{2 \alpha}\right) d s d \xi \\
=\frac{1}{\pi} \int_{0}^{\infty} s^{2 \alpha-1} E_{2 \alpha, 2 \alpha}\left(-\lambda s^{2 \alpha}\right) \int_{0}^{\infty} e^{-s \xi} \cos \xi t d \xi d s=\frac{1}{\pi} \int_{0}^{\infty} E_{2 \alpha, 2 \alpha}\left(-\lambda s^{2 \alpha}\right) \frac{s^{2 \alpha}}{s^{2}+t^{2}} d s
\end{gathered}
$$

This gives Equation (11).

\subsection{Solution Representation}

Theorem 1. Let $\alpha \in(1 / 2,1),|\lambda|>0$, and $|\arg \lambda|<(1-\alpha) \pi-\varepsilon$ for some $\varepsilon>0$. If $f(t) \in C(\mathbb{R}) \cap L^{1}(\mathbb{R})$, then the function

$$
x(t)=\int_{-\infty}^{\infty} f(s) C_{\alpha, \lambda}(t-s) d s
$$

is a regular solution of Equation (7).

Proof. Lemmas 1 and 2 allow us to write

$$
\begin{gathered}
D_{\infty t}^{\alpha} \partial_{-\infty t}^{\alpha} x(t)=\frac{d}{d t} K^{2-2 \alpha} \int_{-\infty}^{\infty} f(s) S_{\alpha, \lambda}^{1}(t-s) d s \\
=\frac{d}{d t} \int_{-\infty}^{\infty} f(s)\left[\frac{\operatorname{sign}(t-s)}{2}-\lambda s_{\alpha, \lambda}^{-1}(t-s)\right] d s=f(t)-\lambda \int_{-\infty}^{\infty} f(s) C_{\alpha, \lambda}(t-s) d s .
\end{gathered}
$$

\section{Conclusions}

The principle of least action is often used to describe dissipative systems, in particular in the case of linear oscillators with viscous friction [26]. In this paper, we used this principle in the context of fractional calculus. In particular, we considered the concept of effective velocity for a moving material point, which was defined in terms of the Gerasimov-Caputo fractional derivative. This allowed us to take into account the heredity and nonlocality in dynamic processes of a material point with respect to the time. Using the fractional Euler-Lagrange equation, we derived the general equation of motion with Gerasimov-Caputo fractional derivatives. The equation of motion of such a form (see Equation (6)), has apparently been obtained for the first time in this paper.

In addition, we expressed the composition of the left and right fractional derivatives with origins at plus and minus infinities in terms of the Riesz potential, and constructed an explicit representation of solutions to a model equation of motion for a fractional oscillator. 
It is of interest to give a detailed physical interpretation for Equations (4) and (6), and apply these equations to real physical processes. Undoubtedly, it is relevant to develop algorithms for the numerical solution of equations in the form of Equation (7). These issues should be the subjects of separate works.

Author Contributions: Conceptualization, L.E., A.P., and S.R.; methodology, L.E., A.P., and S.R.; validation, L.E., A.P., and S.R.; formal analysis, L.E., A.P., and S.R.; investigation, L.E., A.P., and S.R.; writing-original draft preparation, L.E. (Sections 1-5), A.P. (Sections 1, 2, 4, 5), and S.R. (Sections 1-3, 5); writing-review and editing, L.E., A.P., and S.R. All authors have read and agreed to the published version of the manuscript.

Funding: This research received no external funding

Conflicts of Interest: The authors declare no conflict of interest.

\section{References}

1. Tarasov, V.E. Fractional Dynamics: Applications of Fractional Calculus to Dynamics of Particles, Fields and Media; Springer: Berlin/Heidelberg, Germany, 2010.

2. Uchaikin, V.V. Fractional Derivatives for Physicists and Engineers; Springer: Berlin/Heidelberg, Germany, 2012.

3. Herrmann, R. Fractional Calculus-An Introduction for Physicists; World Scientific: Singapore, 2018.

4. Riewe, F. Non-conservative Lagrangian and Hamiltonian mechanics. Phys. Rev. E 1996, 53, $1890-1899$. [CrossRef] [PubMed]

5. Riewe, F. Mechanics with fractional derivatives. Phys. Rev. E 1997, 55, 3581-3592. [CrossRef]

6. Agrawal, O.P. Formulation of Euler-Lagrange equations for fractionalvariational problems. J. Math. Anal. Appl. 2002, 72, 368-379. [CrossRef]

7. Rekhviashvili, S.S. The Lagrange formalism with fractional derivatives in problems of mechanics. Tech. Phys. Lett. 2004, 30, 33-37. [CrossRef]

8. Baleanu, D.; Muslih, S.I. Lagrangian formulation of classical fields within Riemann-Liouville fractional derivatives. Phys. Scr. 2005, 72, 119-121. [CrossRef]

9. Muslih, S.I.; Baleanu, D. Hamiltonian formulation of systems withlinear velocities within Riemann-Liouville fractional derivatives. J. Math. Anal. Appl. 2005, 304, 599-606. [CrossRef]

10. Rabei, E.M.; Nawafleh, K.I.; Hijjawi, R.S.; Muslih, S.I.; Baleanu, D. The Hamilton formalism with fractional derivatives. J. Math. Anal. Appl. 2007, 327, 891-897. [CrossRef]

11. Atanacković, T.M.; Konjik, S.; Pilipovic, S. Variational Problems with Fractional Derivatives: Euler-Lagrange Equations. J. Phys. Math. Theor. 2008, 41, 095201. [CrossRef]

12. Atanacković, T.M.; Stanković, B. On a differential equation with left and right fractional derivatives. Fract. Calc. Appl. Anal. 2007, 10, 139-150. [CrossRef]

13. Atanacković, T.M.; Stanković, B. On a class of differential equations with left and right fractional derivatives. ZAMM Z. Angew. Math. Mech. 2007, 87, 537-564. [CrossRef]

14. Eneeva, L.M. Boundary value problem for differential equation with fractional order derivatives with different origins. Vestn. KRAUNC. Fiz. Mat. Nauk. 2015, 2, 39-44.

15. Eneeva, L.M. On Neumann problem for equation with fractional derivatives with different starting points. Vestn. KRAUNC. Fiz. Mat. Nauk. 2018, 4, 61-65.

16. Eneeva, L.M. Lyapunov inequality for an equation with fractional derivatives with different origins. Vestn. KRAUNC. Fiz. Mat. Nauk. 2019, 3, 32-39.

17. Eneeva, L.M. A priori estimate for an equation with fractional derivatives with different origins. Vestn. KRAUNC. Fiz. Mat. Nauk. 2019, 4, 41-47.

18. Gerasimov, A.N. A generalization of linear laws of deformation and its application to internal friction problem. Prikl. Mat. Mekh. 1948, 12, 251-260.

19. Samko, S.G.; Kilbas, A.A.; Marichev, O.I. Fractional Integrals and Derivatives: Theory and Applications; Taylor \& Francis Books: Didcot, UK, 1993.

20. Nakhushev, A.M. Fractional Calculus and Its Applications; Fizmatlit: Moscow, Russia, 2003.

21. Kilbas, A.A.; Srivastava, H.M.; Trujillo, J.J. Theory and Applications of Fractional Differential Equations; North-Holland Math. Stud.; Elsevier: Amsterdam, The Netherlands, 2006; Volume 204. 
22. Agrawal, O.P. Fractional variational calculus in terms of Riesz fractional derivatives. J. Phys. A: Math. Theor. 2007, 40, 6287-6303. [CrossRef]

23. Rekhviashvili, S.S.; Pskhu, A.V.; Agarwal, P.; Jain, S. Application of the fractional oscillator model to describe damped vibrations. Turk. J. Phys. 2019, 43, 236-242. [CrossRef]

24. Rekhviashvili, S.S.; Pskhu, A.V. New Method for Describing Damped Vibrations of a Beam with a Built-in End. Tech. Phys. 2019, 64, 1237-1241. [CrossRef]

25. Gorenflo, R.; Kilbas, A.A.; Mainardi, F.; Rogosin, S.V. Mittag-Leffler Functions, Related Topics and Applications; Springer: Berlin/Heidelberg, Germany, 2014.

26. Shalashov, A.G. Can we refer to Hamilton equations for an oscillator with friction? Phys. Usp. 2018, 61, 1082-1088. [CrossRef]

Publisher's Note: MDPI stays neutral with regard to jurisdictional claims in published maps and institutional affiliations.

(C) 2020 by the authors. Licensee MDPI, Basel, Switzerland. This article is an open access article distributed under the terms and conditions of the Creative Commons Attribution (CC BY) license (http:/ / creativecommons.org/licenses/by/4.0/). 